\title{
Activation of Human T Cell Clones through the UM4D4/CDw60 Surface Antigen ${ }^{1}$
}

\author{
David A. Fox, ${ }^{*}$ William Davis, ${ }^{*}$ Wendy Zeldes, ${ }^{*}$ Li Kan, ${ }^{*}$ Jay HiggS, ${ }^{*, 2}$ \\ AllaN D. DUBY, $\dagger$ AND JOSEPH HOLOSHITZ $*$
}

*Department of Internal Medicine. Rackham Arthritis Research Unit. and Multipurpose Arthritis Center. University of Michigan, Ann Arbor, Michigan; $\nmid$ Harold C. Simmons Arthritis Research Center, Department of Internal Medicine, University of Texas Southwestern Medical Center, Dallas, Texas; and $\ddagger$ Department of Medicine, Stanford University, Stanford, California

\author{
Received January 8, 1990; accepted March 2, 1990
}

\begin{abstract}
UM4D4 is a recently defined antigen that is expressed on $\sim 25 \%$ of peripheral blood $T$ cells, but on the majority of T cells in inflammatory synovial fluid. Anti-UM4D4 activates peripheral blood $T$ cells in the presence of accessory cells and/or phorbol ester. UM4D4 has been assigned to a new antigen cluster termed CDw60. The present study examined the ability of anti-UM4D4 to activate $\mathrm{T}$ cell clones derived from the synovial fluid of patients with rheumatoid arthritis. UM4D4 was expressed at varying levels on both lectin-generated and antigen-specific clones, including clones of $\mathrm{CD}^{+}, \mathrm{CD}^{+}$, and $\mathrm{CD}^{-}{ }^{-} \mathrm{CD} 8^{-}$phenotypes. Anti-UM4D4 used in soluble form as a single stimulus was typically mitogenic for the $\mathrm{CD} 4^{+}$and some of the $\mathrm{CD} 8^{+}$clones, but not for the $\mathrm{CD}^{-}{ }^{-} \mathrm{CD}^{-}$elones. Phorbol ester boosted the response to anti-UM4D4 in some clones, had no effect in others, and diminished the responses in some cases. In contrast to antiUM4D4, anti-CD3 was generally not mitogenic in soluble form, although it was mitogenic when conjugated to beads. The data show that $\mathrm{T}$ cell clones derived from an inflammatory $\mathrm{T}$ cell infiltrate can be readily activated through the UM4D4/CDw60 antigen. (1) 1990 Academic Press, Inc.
\end{abstract}

\section{INTRODUCTION}

T lymphocyte activation can be induced or augmented by stimulation of a variety of T cell surface structures distinct from the CD3-antigen receptor (CD3-TCR) ${ }^{3}$ complex (1-14). Of these "alternative activation pathways," the best characterized exam-

\footnotetext{
${ }^{1}$ Supported by NIH Grants AR-38477 and AR-39169, a grant from the Michigan Chapter of the Arthritis Foundation, an Arthritis Foundation fellowship award to J. Holoshitz, and the Hulda Irene Duggan Arthritis Investigator Award to D. A. Fox. A. D. Duby is a Pew Scholar in the Biomedical Sciences.

${ }^{2}$ Present address: Wilford Hall USAF Medical Center, Lackland Air Force Base, San Antonio, TX, 78236. The opinions expressed herein are those of the authors and do not necessarily reflect the views of the United States Air Force or the Department of Defense.

${ }^{3}$ Abbreviations used: TCR, T cell receptor; RA, rheumatoid arthritis; PHA, phytohemagglutinin; PMA, tetradecanoyl phorbol acetate; IL-2, interleukin 2; FCS, fetal calf serum; AP-MT, acetone-precipitable fraction, of mycohacterium tuberculosis.
} 
ple in man is CD2 $(1,2)$, for which a natural ligand has been identified, cloned, and implicated as important for $\mathrm{T}$ cell development and activation in vivo (15-17).

Recently we have begun to characterize surface structures that may be important in $T$ cell activation in autoimmune disease by generating monoclonal antibodies against $T$ lymphocyte populations derived from the synovial compartment of patients with rheumatoid arthritis (RA). One such antibody has been termed antiUM4D4 and the surface antigen it binds to is designated UM4D4 (14). UM4D4 is expressed on a minority of peripheral blood $T$ lymphocytes (14), but on the majority of $\mathrm{T}$ cells in inflammatory synovial fluid (18). Although UM4D4 expression is not augmented by $\mathrm{T}$ cell activation in vitro, $\mathrm{T}$ cell clones derived from high-UM4D4 populations maintain expression of this antigen during long-term culture (14). AntiUM4D4 is mitogenic for peripheral blood T cells in the presence of accessory cells and/or phorbol ester, and this response is inhibited by modulation of CD3/TCR (14). It appears, however, that UM4D4 is not closely associated structurally with CD3 or other known $\mathrm{T}$ cell differentiation antigens (14). Immunoprecipitation with antiUM4D4 identified a 92- to 94-kDa structure, following labeling with [ $\left[{ }^{35} \mathrm{~S}\right]$ methionine (19), and a similar band, along with two bands at lower molecular weights, were obtained using Western blotting (14).

Preliminary experiments indicated that anti-UM4D4 was directly mitogenic as a single stimulus for some, but not all, UM4D4 ${ }^{+} \mathrm{T}$ cell clones. The present series of experiments was therefore undertaken to more thoroughly characterize clonal $\mathrm{T}$ cell responses to stimulation through this newly identified activation pathway.

\section{MATERIALS AND METHODS}

\section{TCell Clones}

Two groups of $\mathrm{T}$ cell clones, both derived from RA synovial fluid T cells, were used in these studies. Forty lectin-stimulated clones were generated using PHA, feeder cells, and IL-2 containing medium as previously described $(14,20,21)$. Southern blot analysis of TCR gene rearrangements was used to verify the clonality of these clones (21).

A panel of antigen-specific clones used in these studies has also been previously described (22); these clones were reactive with the acetone-precipitable fraction of mycobacterium tuberculosis (AP-MT).

Expression of surface markers (CD2, CD3, TCR, CD4, CD8, UM4D4) was measured using indirect immunofluorescence and flow cytometry as previously described $(14,22)$.

\section{Activation Assays}

These experiments were performed 11 to 14 days after the last previous restimulation of clones, at a time when ongoing proliferation was negligible. Fifty thousand cloned cells per well were plated in triplicate in 96-well round-bottom plates in RPMI 1640 with $10 \%$ FCS. Stimuli in selected wells included anti-UM4D4 (generally at a 1:100 dilution of ascites), PMA (1 ng/ml), anti-CD3 (OKT3 ascites, 1/100), or antiCD3-Sepharose. Anti-CD3-Sepharose was prepared as previously described (23). After $24 \mathrm{hr}, 0.8 \mu \mathrm{Ci}\left[{ }^{3} \mathrm{H}\right] \mathrm{TdR}$ was added to each well, followed $18 \mathrm{hr}$ later by harvesting 


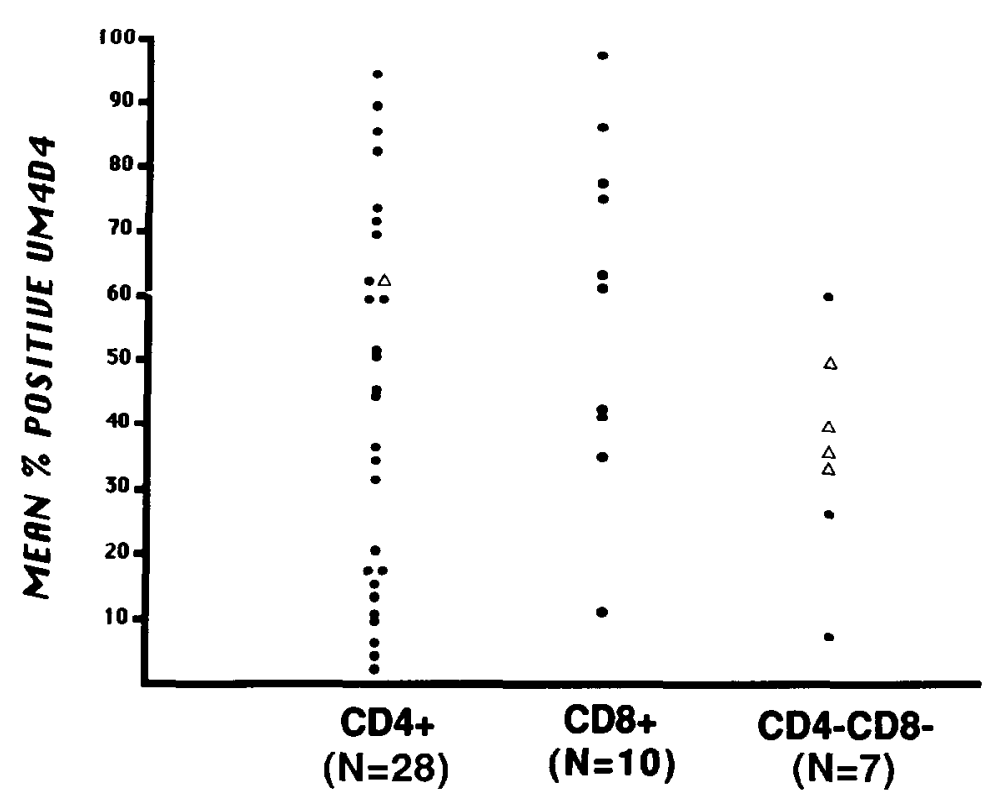

FIG. 1. Expression of UM4D4 (CDw60) on human T cell clones derived from RA synovial fluid, determined by indirect immunofluorescene and flow cytometry using a Coulter Epics $C$ instrument. Lectingenerated clones are indicated by closed circles and antigen-specific clones by open triangles. Each point denotes a separate clone, belonging to the phenotypic subset indicated on the $x$ axis.

of the cultures using a multichannel apparatus, Thymidine incorporation was then quantitated by liquid scintillation counting.

\section{RESULTS}

\section{Expression of UM4D4 on TCell Clones}

Two panels of human $\mathrm{T}$ cell clones derived from RA synovial fluid $\mathrm{T}$ cells were examined. All clones expressed CD2 and CD3. Of 40 lectin-generated clones, 27 were $\mathrm{CD}^{+}, 10 \mathrm{CD}^{+}$, and $3 \mathrm{CD}^{-} \mathrm{CD}^{-}$. All of the lectin-generated clones were $\mathrm{CD} 29^{+} \mathrm{C}-$ $\mathrm{D} 45 \mathrm{R}^{-}$. Four of the five antigen-specific clones used in this study were $\mathrm{CD} 4^{-} \mathrm{CD} 8^{-}$, and these have been shown to express surface $\gamma \delta$ TCR (22). The other antigen specific clone was $\mathrm{CD}^{+}$and it has also been described (22).

Flow cytometric analysis revealed a broad range of UM4D4 expression on both the lectin-stimulated and antigen-specific clones (Fig. 1). Flow cytometry histograms of five representative $\mathrm{CD}^{+}$lectin-generated clones are shown in Fig. 2. Varying degrees of UM4D4 expression were seen within each phenotypic group $\left(\mathrm{CD}^{+}, \mathrm{CD}^{+}\right.$, and $\mathrm{CD}^{-} \mathrm{CD}^{-}$). Unlike antigens such as $\mathrm{CD} 4$ and $\mathrm{CD} 8$, many clones expressed UM4D4 at intermediate levels of fluorescence, with some cells below background intensity, and others strongly positive. This could not be explained by lack of clonality in the populations of cells studied, since monoclonality of all of these clones has been directly verified by Southern blot analysis of TCR gene rearrangements $(21,22)$. The expression of UM4D4 on clones representing the different $T$ cell subsets supports 


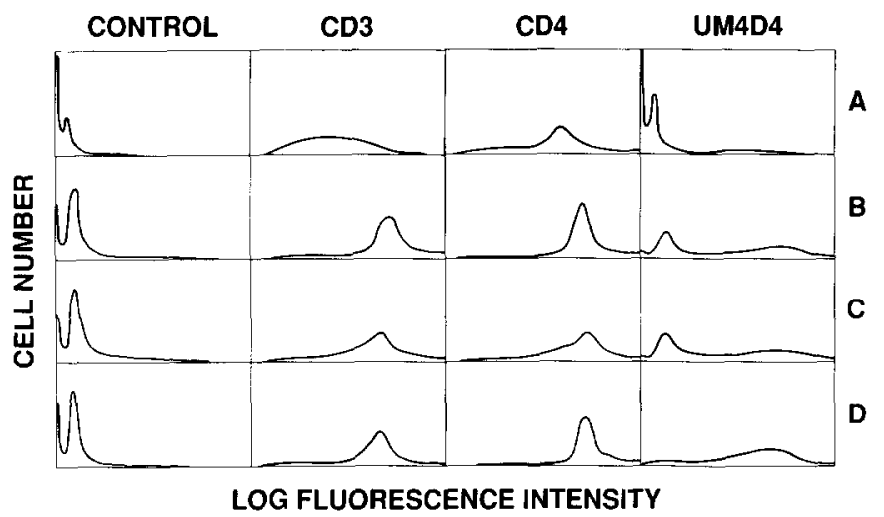

FIG. 2. Flow cytometry histograms of surface antigens expressed by representative $C D 4^{+}$lectin-generated clones. Histograms shown represent background fluorescence, CD3, CD4, and UM4D4. Clonality was verified by Southern blot analysis of TCR gene rearrangements (21). The \% of cells expressing UM4D4 was $8 \%$ for clone A, $50 \%$ for clone B, $45 \%$ for clone C, $85 \%$ for clone D. All clones were $\alpha \beta$-TCR (WT 31 )positive (data not shown).

previous data indicating that UM4D4 defines a phenotypic T cell subset in peripheral blood distinct from populations identified by markers such as CD4 and CD8 (14).

\section{Activation of T Cell Clones by Anti-UM4D4}

Proliferative responses were measured following stimulation of each of the lectingenerated clones by anti-UM4D4. For most clones responses were also measured to soluble anti-CD3, and 10 clones were in addition examined for responses to immobilized anti-CD3 cross-linked to Sepharose beads. As shown in Fig. 3, soluble anti-

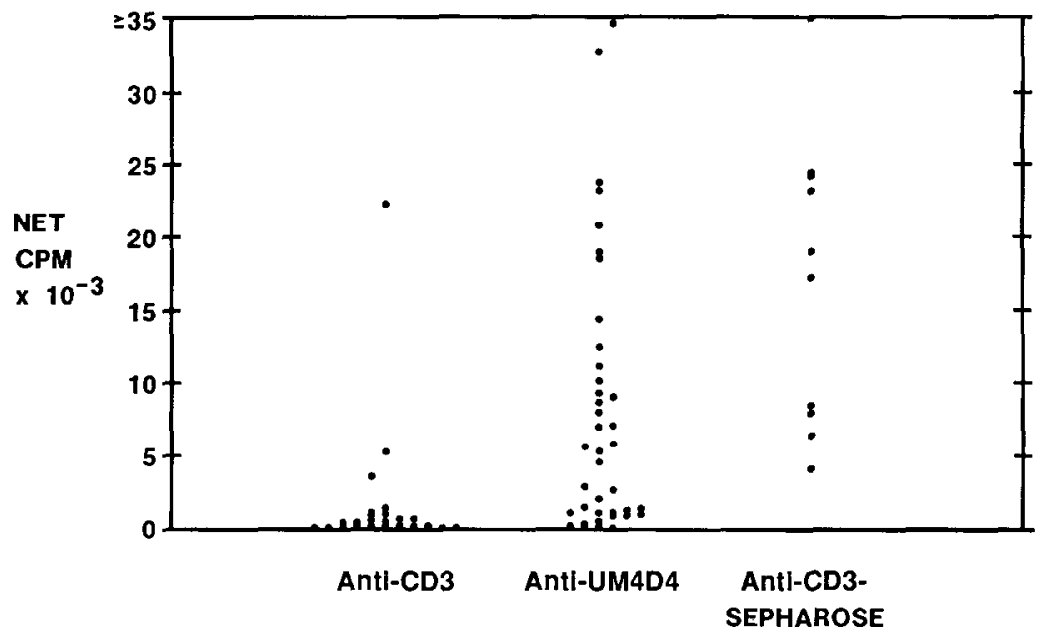

FIG. 3. Proliferative responses of lectin-generated $T$ cell clones derived from RA synovial fluid to soluble anti-CD3, soluble anti-UM4D4, and Sepharose-conjugated anti-CD3. Results are expressed as the mean of triplicate cpm, and each point represents the response of an individual clone to the indicated stimulus. 
TABLE 1

Five Patterns of Clonal T Cell Proliferative Responses to Anti-UM4D4, Observed with Lectin-generated RA Synovial Fluid T Cell Clones

\begin{tabular}{|c|c|c|c|c|}
\hline \multirow[b]{2}{*}{ Group } & \multirow[b]{2}{*}{$(N)$} & \multicolumn{3}{|c|}{ Stimulation index (Mean \pm SEM) } \\
\hline & & Anti-UM4D4 & PMA & Ani-UM4D4 + PMA \\
\hline I & (6) & $1.48 \pm 0.21$ & $1.44 \pm 0.09$ & $1.44 \pm 0.27$ \\
\hline II & (4) & $2.19 \pm 0.36$ & $1.80 \pm 0.30$ & $23.60 \pm 13.90$ \\
\hline III & (10) & $28.66 \pm 7.47$ & $2.89 \pm 0.83$ & $25.28 \pm 5.58$ \\
\hline IV & (9) & $10.46 \pm 2.89$ & $2.34 \pm 0.45$ & $39.23 \pm 10.95$ \\
\hline v & (11) & $73.01 \pm 9.59$ & $3.15 \pm 0.73$ & $25.16 \pm 4.49$ \\
\hline
\end{tabular}

Note. The number of clones in each group is indicated in parentheses. Results are expressed as the mean stimulation index $(\mathrm{cpm} /$ background $\mathrm{cpm}) \pm \mathrm{SEM}$. The stimulation index for each individual clone was calculated from the mean of triplicate $\mathrm{cpm}$.

UM4D4 induced proliferative responses in most clones, varying in magnitude depending upon the clone tested. In contrast anti-CD3 was rarely mitogenic when used in soluble form, although consistently mitogenic when cross-linked to Sepharose. Similar results were obtained when proliferation was measured daily for 3 days following stimulation of selected clones (data not shown).

\section{Heterogeneity of PMA Effects on Responses to Anti-UM4D4}

Since some clones did not respond to anti-UM4D4, experiments were done to determine whether inclusion of an additional costimulatory factor in the culture medium would lead to activation of these clones, and/or alter the responses of clones for which anti-UM4D4 alone was mitogenic. The phorbol ester PMA was used for this purpose at $1 \mathrm{ng} / \mathrm{ml}$, a concentration at which PMA was minimally mitogenic for the clones as a single stimulus. For these experiments a proliferative response was defined as a stimulation index (cpm/background $\mathrm{cpm})>3$, although most responses observed were much stronger.

When the 40 lectin-generated clones were examined in this way, five patterns of responses were observed (Table 1). Clones in group I ( 6 clones) failed to respond to anti-UM4D4, either alone or in the presence of PMA. The 4 clones in group II responded to anti-UM4D4 only when PMA was also present. The majority of clones (30 of 40) responded to anti-UM4D4 alone and are contained in groups III, IV, and $\mathrm{V}$. These latter three groups differed however, in the effect of PMA on the antiUM4D4 response. In group III (10 clones) PMA had no effect on the magnitude of the response. In group IV ( 9 clones) PMA significantly boosted the response. For each clone in this group the response to PMA + anti-UM4D4 was at least 50\% greater than the response to anti-UM4D4 alone, and in general a several-fold increase was observed. The pattern observed in group $\mathrm{V}$ was of particular interest. The eleven clones in this group responded very well to anti-UM4D4 alone and very slightly to PMA alone. In contrast to the other groups, PMA decreased the response of these clones to anti-UM4D4, generally to about one-third and always to < two-thirds of 
TABLE 2

Phenotype of T Cell Clones Belonging to Each Functional Group Delineated in Table 1

\begin{tabular}{rrcccc}
\hline Group & $(N)$ & CD4 pos & CD8 pos & CD4CD8 neg & $\begin{array}{c}\text { \% Pos UM4D4 } \\
\text { (mean } \pm \text { SD) }\end{array}$ \\
\hline I & $(6)$ & 0 & 4 & 2 & $47 \pm 31$ \\
II & $(4)$ & 3 & 0 & 1 & $51 \pm 22$ \\
III & $(10)$ & 7 & 3 & 0 & $40 \pm 27$ \\
IV & $(9)$ & 8 & 1 & 0 & $45 \pm 27$ \\
V & $(11)$ & 9 & 2 & 0 & $50 \pm 30$ \\
\hline
\end{tabular}

Note. The number of clones in each group belonging to the phenotypic subsets defined by CD4 and CD8 expression is indicated. UM4D4 expression is calculated as the mean \pm SD of the $\% \mathrm{UM} 4 \mathrm{D} 4^{+}$cells of all clones in a given group, using flow cytometric data.

the response to anti-UM4D4 alone. When clones were repeatedly tested over a sixmonth period the same pattern of response was seen for a given clone.

Phenotypic characteristics of clones in each group were examined to determine whether surface marker expression correlated with anti-UM4D4 responses. As shown in Table 2 a wide range of UM4D4 expression was found in each group, indicating that some clones with high UM4D4 expression did not respond to anti-UM4D4, and that some clones responded well despite relatively low expression of UM4D4. CD4 ${ }^{+}$ clones all responded to anti-UM4D4 + PMA, and 24 of the $27 \mathrm{CD}^{+}$clones responded to anti-UM4D4 alone. Among $\mathrm{CD}^{+}$clones 6 of 10 responded to antiUM4D4 alone but 4 showed no response to anti-UM4D4 or to anti-UM4D4 + PMA. None of the $3 \mathrm{CD}^{-} \mathrm{CD}^{-}$clones responded to anti-UM4D4 alone, although one showed a weak response to anti-UM4D4 + PMA.

The clones in group $\mathrm{V}$ were studied further because of the unexpected inhibitory effects of phorbol ester on the response to anti-UM4D4. This response pattern did not simply reflect a shift in the kinetics of proliferation in the presence of phorbol ester, since qualitatively similar results were obtained at multiple time points after initiation of the proliferation experiments (data not shown). Effects on the antiUM4D4 response were seen over a range of concentrations of PMA from $0.1-10 \mathrm{ng}$ / $\mathrm{ml}$, although less inhibition of the anti-UM4D4 response was observed at the lowest PMA concentration (Table 3). In addition a comparable decrease in the response to anti-CD3-Sepharose in the presence of PMA was observed in this group of clones, although PMA augmented the response to soluble anti-CD3 (Table 3). In contrast to the effects of PMA, costimulation with recombinant IL-2 did not reduce the response to anti-UM4D4 (data not shown).

\section{Effects of Anti-UM4D4 on Antigen-Specific Clones}

The activation experiments described above were done with lectin-generated clones. Additional experiments were performed to determine whether anti-UM4D4 was also mitogenic for $\mathrm{UM}_{4} 4^{+}$clones generated and maintained by stimulation with a specific antigen, AP-MT.

A representative TCR $\alpha \beta$ clone (Table 4) responded either to specific antigen or to anti-UM4D4. In contrast, two $\mathrm{CD} 4^{-} \mathrm{CD}^{-} \gamma \delta$ clones, which also responded to spe- 
TABLE 3

Effect of PMA on Responses of group V Clones to Anti-UM4D4 and Anti-CD3

\begin{tabular}{lrccccc}
\hline & \multicolumn{7}{c}{ PMA (ng/ml) } \\
\cline { 2 - 7 } \multicolumn{1}{c}{ Stimuli } & 0 & 0.1 & 0.5 & 1 & 5 & 10 \\
\hline None & $348 \pm 107$ & $1116 \pm 236$ & $1447 \pm 296$ & $1498 \pm 388$ & $1690 \pm 571$ & $1656 \pm 495$ \\
Anti-UM4D4 & $27149 \pm 6151$ & $13423 \pm 2295$ & $12004 \pm 2254$ & $8151 \pm 1601$ & $6493 \pm 1084$ & $6951 \pm 1884$ \\
Anti-CD3-Sepharose & $35704 \pm 5623$ & N.D. & N.D. & $7259 \pm 1621$ & N.D. & N.D. \\
Anti-CD3 & $1913 \pm 957$ & N.D. & N.D. & $6497 \pm 3175$ & N.D. & N.D. \\
\hline
\end{tabular}

Note. Four representative clones were studied. The results are expressed as the mean \pm SEM of the responses for all four clones. For each clone wells were set up in triplicate, and the mean cpm in each group was calculated.

cific antigen, failed to respond to anti-UM4D4 (Table 4). One clone was tested with anti-UM4D4 + PMA, and showed only minimal proliferation to this combination of stimuli. All three clones responded to soluble anti-CD3 in the presence of added accessory cells.

\section{DISCUSSION}

In view of the strong expression of UM4D4/CDw60 on $T$ cells found in certain compartmentalized autoimmune conditions, characterization of $\mathrm{T}$ cell activation through this structure is of particular interest. In this study $\mathrm{T}$ cell clones derived from RA synovial fluid were used since almost all of them express UM4D4, although at varying levels. The data indicate that a wide range of UM4D4 expression is found on clones within each of the phenotypic subsets defined by CD4 and CD8 (CD4 ${ }^{+}, \mathrm{CD}^{+}$, and $\mathrm{CD} 4^{-} \mathrm{CD}^{-}$). The lectin-stimulated $\mathrm{CD} 4^{-} \mathrm{CD} 8^{-}$clones, in contrast to the $\mathrm{CD} 4^{+}$ and $\mathrm{CD} 8^{+}$clones, showed no staining with the WT31 monoclonal antibody directed against TCR $\alpha \beta$ (data not shown). Although antibodies against TCR $\gamma \delta$ were not available during the period of viability of these clones, it is highly likely that these were TCR $\gamma \delta$ clones. The TCR components of the antigen-specific clones used in

TABLE 4

Responses of AP-MT-Specific Clones to Stimulation by Antigen or by Anti-UM4D4

\begin{tabular}{lrrr}
\hline & \multicolumn{3}{c}{ Clone } \\
\cline { 2 - 4 } \multicolumn{1}{c}{ Stimuli } & $1.1(\alpha \beta)$ & $1.2(\gamma \delta)$ & $1.4(\gamma \delta)$ \\
\hline None & $882 \pm 90$ & $367 \pm 311$ & $1209 \pm 175$ \\
AP-MT & $7563 \pm 307$ & $4449 \pm 610$ & $5633 \pm 294$ \\
Anti-UM4D4 & $5459 \pm 757$ & $175 \pm 8$ & $1493 \pm 21$ \\
Anti-UM4D4 + PMA & N.D. & $1431 \pm 278$ & N.D. \\
\hline
\end{tabular}

Note. T cell clones were cultured in the presence of accessory cells as previously described (22). The TCR expressed by each clone is indicated in parentheses. Results are expressed as the mean \pm SEM of triplicate cpm. 
these studies have been fully characterized (22), and it is therefore clear that UM4D4 can be expressed on clones bearing TCR $\gamma \delta$, as well as on clones bearing TCR $\alpha \beta$. The mechanisms regulating the surface intensity of UM4D4 expression are not yet clear, but the current and previously published (14) data indicate that expression is heterogeneous on populations that are unequivocally clonal, to an extent not seen with conventional $T$ cell subset markers such as CD4 and CD8. The fluorescence pattern observed for individual clones was generally stable on repeat testing over intervals of 2-6 months, in contrast to the instability of UM4D4 expression observed on malignant $\mathrm{T}$ cell lines $(14,19)$.

The current experiments demonstrate that anti-UM4D4 can potently activate T cell clones, even when used as a single stimulus, in the absence of additional factors such as accessory cells, IL-2, or phorbol ester. Feeder cells are no longer detectable in the clone cultures at the time interval after prior restimulation during which antiUM4D4 responses were tested. The absence of accessory cells is supported by the general lack of response of the clones to soluble anti-CD3, in contrast to Sepharoseconjugated anti-CD3 which, as expected, was consistently mitogenic. The findings indicate that for $T$ cell clones, the activation requirements for UM4D4 pathway triggering differ from the requirements for activation through the CD3-TCR complex. Anti-UM4D4 is an IgM antibody, in contrast to the IgG isotype of the mitogenic anti-CD3 antibody used in these studies. This discrepancy does not, however, explain the different effects on clonal proliferation, since a prototypic IgM anti-CD3 antibody is nonmitogenic and inhibits various $T$ cell responses $(14,24,25)$.

The effects of anti-UM4D4 are similar to responses observed in murine cells with antibodies directed against the Thy- 1 and TAP antigens $(9,12)$, but available biochemical information about UM4D4 $(14,19)$ does not suggest any similarity to Thy1 or TAP.

It should be noted that in contrast to clones, peripheral blood $\mathrm{T}$ cells cannot be activated by anti-UM4D4 in the absence of accessory cells unless a second stimulus, such as PMA, is added (14). Even in the presence of monocytes, responses may be suboptimal without PMA (14). These findings imply that prior activation of $T$ cells could render them more susceptible to activation by anti-UM4D4, even though UM4D4 expression does not appear to increase as a consequence of $T$ cell activation (14).

Interesting heterogeneity was observed in the clonal responses to anti-UM4D4. Some clones did not proliferate in the presence of anti-UM4D4, although costimulation with anti-UM4D4 + phorbol ester induced proliferation in 6 of 10 of these clones (group II). Lack of response could not be explained by lower expression of UM4D4. Interestingly, all of the $\mathrm{CD} 4^{-} \mathrm{CD} 8^{-}$clones tested either failed to respond to antiUM4D4 under any circumstances, or else showed a very minimal response in the presence of PMA.

Striking heterogeneity was also observed in the clones that did respond to antiUM4D4, with phorbol ester reproducibly either increasing, not affecting, or lowering the response of a given clone. In group $\mathrm{V}$, in which inhibitory effects of PMA were observed, a similar inhibition of responses to anti-CD3-Sepharose was also seen. This could not be explained simply by toxic effects of PMA on these clones since, the number and viability of cells was not reduced, the effects were observed at PMA concentrations as low as $0.1 \mathrm{ng} / \mathrm{ml}$, and the response to soluble anti-CD3 was en- 
hanced rather than lowered. The findings imply that under certain circumstances activation (or perhaps excessive activation) of protein kinase $C$ in $T$ cell clones can exert growth-inhibitory rather than stimulatory effects. Similar inferences have been drawn from observations of phorbol ester effects in other systems (26). Possible effects of PMA other than activation of protein kinase $\mathrm{C}$ are, however, difficult to exclude. It is also of interest that activation of protein kinase $C$ in a murine $T$ cell line by diacyglycerol analogs, but not PMA, was found to limit cell activation and IL-2 production (27).

The results of these studies raise questions about the signaling mechanisms employed by the UM4D4 activation pathway, and suggest that heterogeneity in signaling might be found in different clones, or in clones compared to peripheral blood $T$ cells. Preliminary findings (Fox, D. A., Davis, W., June, C., unpublished observations) indicate that peripheral blood $T$ cells can proliferate in response to anti-UM4D4 in the absence of a detectable increase in cytoplasmic free calcium. Further analysis of several potential signaling mechanisms will therefore be required to resolve these issues.

The results obtained using clones specific for the AP-MT antigen indicate that repetitive stimulation with antigen is compatible with continued expression of UM4D4, and in certain cases with retention of the capacity to be activated through UM4D4. This suggests that, if a ligand of UM4D4 were present in autoimmune lesions such as RA synovial tissue, the UM4D4 pathway could contribute to maintaining activation of $\mathrm{T}$ cells that had initially responded to specific antigen.

\section{ACKNOWLEDGMENTS}

We thank Clare Rogers for performing the flow cytometry, and Marcia Bauer for careful preparation of the manuscript.

\section{REFERENCES}

1. Meuer, S. C., Hussey, R. D., Fabbi, M., Fox, D. A., Acuto, O., Fitzgerald, K. A., Hodgdon, J. D., Protentis, J. P., Schlossman, S. F., and Reinherz, E. L., Cell 36, 897, 1984.

2. Fox, D. A., Hussey, R. E., Fitzgerald, K. A., Bensussan, A., Daley, J. F., Schlossman, S. F., and Reinherz, E. L., J. Immunol. 133, 330, 1985.

3. Hara, T., Fu, S. M., and Hansen, J. A., J. Exp. Med. 162, 1513, 1985.

4. Moretta, A., Pantaleo, G., Polez-Botet, M., and Moretta, L., J. Exp. Med. 162, 823, 1985.

5. Fleischer, B., J. Immtunol. 138, 1346, 1987.

6. Mentzer, S. J., Remold-O’Donnell, E., Crimmins, M. A. V., Bierer, B. E., Rosen, F. S., and Burakoff, S. J., J. Exp. Med. 165, 1383, 1987.

7. Carrel, S., Isler, P., Salvi, S., Giuffre, L., Pantaleo, G., Mach, J. P., and Cerottoni, J. C., Eur. J. Immunol. 17, 1395, 1987.

8. Carrel, S., Salvi, S., Giuffre, L., Isler, P., and Cerottini, J. C., Eur. J. Immunol. 17, 835, 1987.

9. Gunter, K. C., Malek, T. R., and Shevach, E. M., J. Exp. Med. 159, 716, 1984.

10. Pircher, H., Groscurth, P., Baumhutter, S., Aguet, M., Zinkernagel, R. M., and Hengartner, H., Eur. J. Immunol. 16, 172, 1986.

11. Malek, T. R., Ortega, G., Chan, C., Kroczek, R. A., and Shevach, E. M., J. Exp. Med. 164, 709, 1986.

12. Rock, K. L., Yeh, E. T. H., Gramm, C. F., Haber, S. I., Reiser, H., and Benacerraf, B., J. Exp. Med. $163,315,1986$.

13. Isler, P., Salvi, S., Rapin, C., Guiffre, L., Cerottini, J. C., and Carrel, S., Eur. J. Immunol. 18, 1491, 1988.

14. Higgs, J. B., Zeldes, W., Kozarsky, K., Schteingart, M., Kan, L., Bohlke, P., Krieger, K., Davis, W., and Fox, D. A., J. Immunol. 140, 3758, 1988. 
15. Selvaraj, P., Plunkett, M. L., Dustin, M., Sanders, M. D., Shaw, S., and Springer, T. A., Nature (London) 326, 400, 1987.

16. Seed, B., Nature (London) 329, 840, 1987.

17. Vollger, L. W., Tuck, D. T., Springer, T. A., Haynes, B. F., and Singer, K. H., J. Immunol. 138, 358, 1987.

18. Fox, D. A., Millard, J. A., Kan, L., Zeldes, W. S., Davis, W., Higgs, J. B., Emmrich, F., and Kinne, R., submitted for publication, 1989.

19. Fox, D. A., Kan, L., Chan, L. S., Baadsgaard, O., Cooper, K. D., and Kozarsky, K., "Leukocyte Typing IV." (W. Knapp, B. Dorken, W. R. Gilks, E. P. Rieber, R. E. Schmidt, H. Stein, and A. E. G. Kr. von dem Borne, Eds.), pp. 364-366. Oxford Univ. Press, Oxford, 1990.

20. Moretta, A., Pantaleo, G., Moretta, L., Mingari, M. C., and Cerottini, J. C., J. Exp. Med. 158, 571, 1983.

21. Duby, A. D., Sinclair, A. K., Osborne-Lawrence, S. L., Zeldes, W., Kan, L., and Fox, D. A., Proc. Natl. Acad. Sci. USA 86, 6206, 1989.

22. Holoshitz, J., Koning, F., Coligan, J. E., DeBruyn, J. D., and Strober, S., Nature (London) 339, 226, 1989.

23. Meuer, S. C., Hodgdon, J. C., Hussey, R. E., Protentis, J. P., Schlossman, S. F., and Reinherz, E. L., J. Exp. Med. 158, 988, 1983.

24. Reinherz, E. L., Meuer, S., Fitzgerald, K. A., Hussey, R. E., Levine, H., and Schlossman, S. F., Cell 30, 735, 1982.

25. Fox, D. A., Schlossman, S. F., and Reinherz, E. L., J. Immunol. 136, 1945, 1986.

26. Nishizuka, Y., Science 233, 305, 1986.

27. Mills, G. B., May, C., Hill, M., Ebanks, R., Roifman, C., Mellors, A., and Gelfand, E. W., J. Immunol. 142, $1995,1989$. 